\title{
Agôn
}

Revue des arts de la scène

$4 \mid 2011$

L'objet

\section{Le décor comme objet dans l'esthétique théâtrale irrégulière du XVIIe siècle}

\section{Marc Bayard}

\section{(2) OpenEdition \\ Journals}

Édition électronique

URL : http://journals.openedition.org/agon/2024

DOI : 10.4000/agon.2024

ISSN : 1961-8581

Éditeur

Association Agôn

Référence électronique

Marc Bayard, « Le décor comme objet dans l'esthétique théâtrale irrégulière du XVIle siècle », Agôn [En ligne], 4 | 2011, mis en ligne le 14 janvier 2012, consulté le 17 avril 2020. URL : http:// journals.openedition.org/agon/2024; DOI : https://doi.org/10.4000/agon.2024 


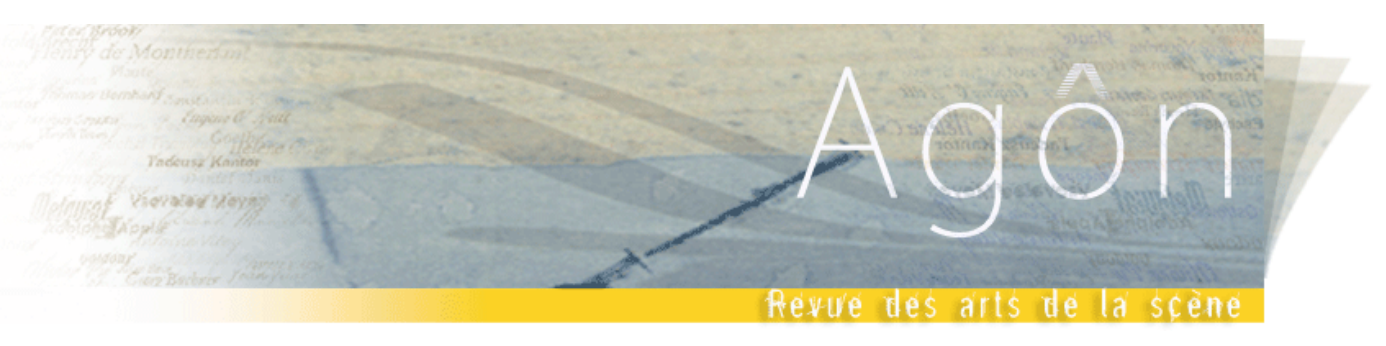

Le décor comme objet dans l'esthétique théâtrale irrégulière du XVIIe siècle

Marc Bayard

Le Mémoire de Laurent Mahelot (v. 1620-1640), avec les dessins de Georges Buffequin (v. 1586-1641) et les listes d'accessoires des pièces qui accompagnent ces esquisses, propose une vision originale de l'esthétique théâtrale irrégulière et de son utilisation des objets.

Cette esthétique de la variété confère aux décors et aux objets une dimension particulière, performative, que l'on ne retrouve pas dans l'écriture régulière dite classique. Au delà de ces "décors-objets", ce théâtre déploie également des objets que l'on pourrait qualifier d' "immatériels-réels", une catégorie singulière où l'aspect solide propre à l'objet est remis en question.

Le XVIIe siècle offre de multiples révolutions dans le domaine des sciences et des arts. Ces changements en profondeur atteignent notamment l'art poétique et théâtral français au milieu du siècle, en particulier à la suite de la Querelle du Cid et autour de la question de la vraisemblance ${ }^{1}$. La mise en place d'une esthétique de l'unité poétique, en droite ligne d'une application des conceptions aristotéliciennes redécouvertes en Italie puis en France à la Renaissance, s'opère au détriment d'une esthétique de la pluralité, également redécouverte en Italie mais qui a eu plus d'impact en France à la fin du XVIe et au début du XVIIe siècle ${ }^{2}$. Un basculement épistémologique intervient ainsi dans les années 1630-1650, décennies qui voient

1 Je remercie les directrices du dossier, Émilie Charlet, Aurélie Coulon et Anne-Sophie Noel, pour leur relecture attentive, ainsi que l'équipe de la revue Agôn.

Giovanni Dotoli, Temps de préfaces : le débat théâtral en France de Hardy à la querelle du "Cid", Paris, Klincksieck, 1997; Jean-Marc Civardi, La querelle du Cid (1637-1638), Paris, Champion, 2004 ; Emmanuelle Hénin [dir.], Les Querelles dramatiques à l’Âge classique (XVIIeXVIIIe siècles), Louvain, Peeters, coll. La République des Lettres 40, 2010 et Anne Duprat, Vraisemblances. Poétiques et théorie de la fiction du Cinquecento à Jean Chapelain (1500-1670), Paris, Champion, 2009. Sur la question de la théorie théâtrale au XVIIe siècle, voir notamment: Emmanuelle Hénin, Ut pictura theatrum: théâtre et peinture de la Renaissance italienne au classicisme français, Genève, Droz, 2003; Déborah Blocker, Instituer un "art": politiques du théâtre dans la France du premier XVIIe siècle, Paris, Champion, 2009.

2 Sur la pensée d'Epicure et de Lucrèce, voir notamment: Simone Fraisse, Une conquête du rationalisme, l'influence de Lucrèce en France au XVIe siècle, Paris, Nizet, 1962 ; Susanna Gambino Longo, Savoir de la nature et poésie des choses. Lucrèce et Épicure à la Renaissance italienne, Paris, Champion, 2004 ; Frank Lestringant [dir.], La renaissance de Lucrèce, Paris, PU Paris-Sorbonne, Collection Cahiers V.L. Saulnier, 2010. 
triompher un art de l'unité (l'esthétique régulière), au détriment d'une pratique de la poétique de la variété qui voulait se libérer des conceptions aristotéliciennes (l'esthétique irrégulière) ${ }^{3}$.

Ce basculement intervient précisément au théâtre de l'Hôtel de Bourgogne, à Paris, reconnu par la monarchie dès le début du XVIIe siècle. Un document, le Mémoire de Laurent Mahelot ${ }^{4}$, révèle avec précision l'activité et l'évolution scénographique de ce lieu. J'ai démontré dans des études précédentes que les quarante-sept dessins (sur les soixante-et-onze pièces et leur liste d'accessoires) qui composent la première partie de ce manuscrit ont été réalisés par Georges Buffequin (v. 1585-1641) ${ }^{5}$ dans les années 1620-1640. Peintre du roi au service de Richelieu au palais cardinal et scénographe dans un théâtre public (l'Hôtel de Bourgogne), Buffequin, par ses esquisses à la mine et au lavis, et avec la liste des accessoires et des décors qui les accompagnent, propose différents décors que l'on peut regrouper en quatre catégories formelles: les éléments végétaux (bois, jardins, arbres, végétations) et naturels (antres, rochers, montagnes), associés à des architectures (fontaines, maisons appelées rustiques) et des architectures comportant des éléments architecturaux indépendants (façade de palais, tours, prisons, chambres, façade de maisons - cf. tableau ci-dessous) ${ }^{6}$. Au-delà de ces aspects formels, les œuvres de Buffequin témoignent des recherches d'une nouvelle esthétique qui secoue le monde théâtral durant le règne de Louis XIII ${ }^{7}$, même si le maître des feintes n'est probablement pas l'auteur conceptuel de ces évolutions.

À l'intérieur du manuscrit du Mémoire de Laurent Mahelot, on repère la succession historique de la composition irrégulière à l'écriture régulière. L'art régulier, qui triomphe dans les années 1640 , visible déjà en partie dans les dessins de Buffequin $^{8}$ et dans les spectacles pour Richelieu (la soirée d'inauguration de Mirame en $1641^{9}$ ), rationalise les unités de temps, d'action et de lieu mais il développe aussi

3 Marc Bayard, « De la cohérence à la cohésion : quelques réflexions sur la construction de la théorie de l'unité dans les arts en Italie et en France (XVIe-XVIIe siècles) », Marc Bayard [dir.], Rome-Paris, 1640. Transferts culturels et renaissance d'un centre artistique, Rome/Paris, AFR/Somogy, 2010, p. 415-431.

$4 \quad$ Pierre Pasquier (éd.), Le Mémoire de Mahelot, Laurent et d'autres décorateurs de l'Hôtel de Bourgogne et de la Comédie-Française au XVIIe siècle, Paris, Champion, 2005.

5 Marc Bayard, «Les faiseurs d'artifices : Georges Buffequin et les artistes de l'éphémère à l'époque de Richelieu », Revue XVIIe siècle, $\mathrm{n}^{\circ} 230,1-2006$, p. 151-164; id., «Les dessins du Mémoire de Laurent Mahelot: sur les traces d'un peintre du roi au service de Richelieu », Revue d'histoire $d u$ théâtre, 2006-4, p. 313-324, id., "Richelieu en scène, Buffequin en coulisse », Richelieu, patron des arts, J.-C. Boyer, B. Gaehtgens et B. Gady [dir.], Paris, Éditions de la Maison des Sciences de 1'homme, 2009, p. 475-500.

$6 \quad$ NDLE : ce tableau intitulé «Le Mémoire de Laurent Mahelot: tableau comparatif des différents décors et accessoires » est placé en "documents annexes" ci-dessous et consultable en fichier pdf.

7 Marc Bayard, Feinte Baroque. Iconographie et esthétique de la variété au XVIIe siècle, Marc Bayard, Rome/Paris, Académie de France à Rome/Somogy, 2010.

$8 \quad$ Par exemple le dernier dessin du Mémoire de Laurent Mahelot avec Les Vendanges de Suresnes de Pierre du Ryer.

$9 \quad$ La Gazette de France du 19 janvier 1641 rapporte à propos de la représentation de Mirame : «Après la comédie circonscrite par les loix de la poësie dans les bornes de ce jour naturel : les nuages d'une toile abaissée cachèrent entierement le théâtre. [...] \& peu après sortit de dessous cette toile un pont doré conduit par deux paons qui fut roulé depuis le théâtre jusques sur le bord de 
une économie de moyens où se réduit l'utilisation des objets sur scène. De la sorte, les nouvelles pratiques durant cette période ne se situent pas uniquement dans l'écriture: les expérimentations interviennent également dans l'utilisation des éléments scénographiques, en particulier dans celui des objets.

Pour interroger cette notion, il est intéressant d'examiner avec attention l'esthétique irrégulière que nous proposent les œuvres graphiques et les accessoires de l'Hôtel de Bourgogne. Si Buffequin emploie avec prodigalité des objets matériels que nous examinerons dans un premier temps, il utilise également, de façon inattendue, des objets que l'on pourrait qualifier d'immatériels, notion bien évidemment ambiguë qu'il s'agit à la fois de mettre en exergue et de circonvenir avec précision. Notre approche est celle de l'historien de l'art : l'aire dramatique y est en effet examinée comme une image (le décor) dont la caractéristique principale est d'être disposée dans un espace ; une tridimensionnalité de l'image du décor. Ces deux entités constituent un enjeu historique, aussi bien dans la constitution formelle (son élaboration au cours du temps), que dans ses dimensions théoriques (les arts poétiques, les esthétiques de la machinerie et les conceptions spatiales). L'histoire de l'art focalise ainsi son regard sur les aspects historiques d'une forme et sa matérialité (peinture, sculpture, décor... ou des objets scéniques), pour en saisir les résonances et les perspectives historiques: l'iconographie pour apprécier l'iconologie. En ce qui concerne le sujet qui nous préoccupe, les objets scénographiques, l'attention sera plus particulièrement portée sur une délimitation formelle, pas toujours simple à opérer, et sur une tentative d'ouvrir ces démarcations, ceci grâce à un éclairage historique plus large.

Pour débuter, il n'est pas inutile de poser la définition habituelle de l'objet. Il s'agit en effet d'une "Chose solide, maniable, généralement fabriquée, une et indépendante, ayant une identité propre, qui relève de la perception extérieure, elle appartient à l'expérience courante et répond à une certaine destination ${ }^{10}{ }$. Si la notion d'objet solide, comme je vais le montrer dans un premier temps, recoupe bien les éléments scéniques utiles à la dramaturgie de Buffequin, j'indiquerai également qu'il est possible d'élargir cette notion d'objet à des entités non solides, à une catégorie que l'on qualifiera d'immatérielle, mais qui est également «maniable, généralement fabriquée, une et indépendante, ayant une identité propre, qui relève de la perception extérieure, appartient à l'expérience courante et répond à une certaine destination ». De la sorte, l'objet utilisé dans la scénographie irrégulière n'a pas pour seul critère invariant la notion de solidité. La notion d'objet est ici utilisée selon son premier degré, empirique, observable et délimité, et ne recoupe pas la notion d'objet au champ d'investigation plus large, telle qu'elle a pu l'être par exemple dans le schéma actantiel de Greimas ${ }^{11}$ : l'objet n'est pas ici un objectif,

l'eschaffaut de la Reine, \& aussitost la toile se leva, \& au lieu de tout ce qu'avoit esté veu sur le théâtre, y parut une grande salle en perspective, dorée et enrichie des plus magnifiques ornemens, éclairée de seize chandeliers de cristal» (c'est nous qui soulignons). Sur cette soirée, voir : Marc Bayard, «Le roi au cœur du théâtre : Richelieu met en scène l'Autorité », L'image du roi de François Ier à Louis XIV, Thomas W. Gaehgtens et Nicole Hochner [dir.], Actes de colloque 2001, Centre Allemand d'histoire de l'art/Éditions de la Maison des sciences de l'homme, Paris, 2006, p. 191-208.

10 Dictionnaire CNRTL : URL : http://www.cnrtl.fr/definition/ [site consulté le 31/10/2011].

11 Algirdas Julien Greimas, Sémantique structurale : recherche et méthode, Paris, Larousse, 1966. 
mais bien une structure utile au mouvement sur scène et au déroulé poétique. Une entité identifiable, observable, qui possède des limites dans le temps et dans son processus d'émergence, mais qui prend une signification poétique ou dramaturgique, et qui se charge d'un sens compréhensible par le spectateur au moment où il est activé par le jeu des comédiens et le déroulé de l'action. Cet objet peut être visible sur scène mais sans posséder une charge sémantique déterminée jusqu'à son activation par la valeur langagière ou cinétique de l'acteur.

L'article propose donc d'examiner la notion d'objet scénique de l'esthétique théâtrale irrégulière selon une signification traditionnelle liée à la matérialité d'une forme servant le jeu du comédien. Mais aussi, dans une extension sémantique, par l'observation d'objet comme étant des entités spécifiques, utilisées d'une manière ou d'une autre par la scénographie et le jeu des comédiens, qui toutefois n'ont pas la délimitation matérielle et solide spécifique de la première catégorie.

\section{L'objet matériel dans l'esthétique irrégulière}

\section{L'objet conjoncturel : l'accessoire}

Il faut, au milieu du theatre, un pallais dans un jardin, ou il y ayt deux fenestres grillees et deux escaliers ou il y a des amants qui se parlent. A un des bouts du theatre, une fontaine dans un bois et, de l'autre costé, une ruine dans un bois. Au premier acte, il faut des rossignols. Au troisiesme et au cinquiesme acte, l'on faict paraistre une nuict, une lune et des estoilles. Des rondaches, des dards, des fleurets ; des flambeaux de cire avec des flambeaux d'argent ou autres, il n'importe. Il faut aussy une bague d'or et une mandille de lacquais ${ }^{12}$.

La liste des accessoires des Occasions perdues de Jean de Rotrou (fig. 1), un exemple parmi tant d'autres du Mémoire de Laurent Mahelot, donne une idée de l'intérêt de ces notes manuscrites qui sont jointes au dessin de Buffequin. Un autre exemple précise l'intérêt de cette liste au regard de la notion d'objet théâtral :

$\mathrm{Au}$ milieu du theatre, un temple fort superbe, qui sert au 5me acte, est le plus beau du theatre, enrichy de lierre, d'or clinquant, balustres, termes ou colomnes, un tableau de Diane au milieu de l'autel, deux chandeliers garnis de chandelles. A un costé du theatre, il faut une prison en tour ronde ; que la grille soit fort grande et basse pour voir trois prissonniers. A costé de la prison, il faut un beau jardin spacieux orné de ballustres, de fleurs et de pallissades. De l'autre costé du theatre, il faut une montaigne eslevée ; sur ladicte montaigne, un tombeau, un pilier, un carquan, et un autel boccager de verdure et rocher, ou l'on puisse monter sur ledict rocher devant le peuple. A costé du rocher, un antre, une mer, un demy vaisseau. Sous le rocher, faire paraistre une prison pour deux personnes, qui soit cachée. Il faut du sang, des esponges, une petite peau pour faire la feinte du cou du sacrificateur, un chapeau de fleurs, un flambeau de cire. Il se fait une nuict, si l'on veut. Il faut des turbans pour des Turcs, des dards, des javelots, tambours, trompettes, des chesnes, des clefs, une

12 Occasions perdues, tragi-comédie de Jean de Rotrou, dans Le Mémoire de Laurent Mahelot, Bnf, Ms. fr. 24330, f. 10v. Cité dans Marc Bayard, Feinte baroque..., op. cit., p. 196. 
robe de conseiller, deux bourguinottes, de la verdure, une lanterne sourde et une chandelle dedans ${ }^{13}$. (fig. 2)

Ces deux listes montrent la richesse du Mémoire de Laurent Mahelot. Dès la première lecture, il apparaît, dans la manière de conduire la liste, une différence entre les décors proprement dits (un palais avec un jardin, des fenêtres praticables par les comédiens, une fontaine dans un bois, un temple, une prison...), les objets scéniques utiles à l'action singulière des acteurs (rondaches, dards, fleurets, flambeaux d'argent, une bague, rossignols, javelots, vêtements, clefs...), et, enfin, les effets scénographiques. Dans l'économie globale de l'énumération de la liste, le maître des feintes n'établit pas clairement de distinction entre les différents éléments de chaque scénographie. En revanche, il commence généralement par évoquer la liste des décors et il débute l'énumération par le milieu de la scène («il faut au milieu du théâtre...», un palais, un temple...). Il expose ensuite les différents compartiments de part et d'autre du centre de l'aire de jeu et il énonce les effets éventuels («l'on fait paraistre une nuict», «relever le rideau », «faire paraître un char »...). Enfin, Buffequin annonce les accessoires utiles au jeu des comédiens. Il part donc de l'aspect structurant du décor pour aboutir au conjoncturel scénographique. Il y a ainsi une distinction non énoncée entre le décor, qui encadre l'action, et l'objet de plus petite dimension. Mais à aucun moment il n'y a clairement une différentiation entre l'accessoire et l'objet. On aurait même tendance à penser que tous les éléments de la scénographie sont des objets, mais que chacun comporte des usages distincts. Le décor souligne la catégorie poétique de la pièce (le décor végétal pour la pastorale, le décor urbain pour la comédie ou la tragédie), même si ces catégories, avant le règne de la doctrine classique de la règle des unités, semblent bien difficiles à stabiliser. Tandis que l'objet de plus petite dimension caractérise plutôt l'action du comédien.

L'étude des dessins de Buffequin, en même temps que la liste des accessoires, offre une vision de la mise en scène et de l'image du décor dans la première partie du XVIIe siècle. Il est possible de la sorte de posséder une idée synthétique de l'esthétique théâtrale des années 1630 à Paris. Des objets sont utilisés sur scène par les comédiens pour accompagner, préciser ou prolonger l'action dramatique, et l'emploi d'armes, de bijoux, de vaisselles, d'outils divers signalent de cette manière l'action de l'acteur ou son statut social. Toutefois, ce qui singularise les décors de Buffequin est l'usage parfois très particulier non pas des objets mais des décors. Les feintes acquièrent ainsi le statut d'objet scénique car, très visibles sur scène, elles ont en revanche une utilisation scénique plutôt réduite : leur présence à la vue du spectateur est importante, mais leur utilisation dramatique est réduite, ce qui rapproche les décors des objets scéniques de plus petite dimension.

\section{Le décor irrégulier comme objet structurel}

Nous distinguerons deux types de décor. Nous avons d'un côté le "décordécorum", né dans les années 1640, qui prend sa source dans une perspective qui unifie les formes: cette feinte est un lieu neutre, décorum de l'action, image rhétorique qui accompagne le discours et qui vient donner un cadre visible et

13 Clitophon, tragi-comédie de Pierre du Ryer, dans Le Mémoire de Laurent Mahelot, Bnf, Ms. fr. 24330, f. 47v. Cité dans Marc Bayard, Feinte baroque..., op. cit., p. 218. 
spécifique à l'action dramatique. Il est neutre car il est une résonance, une amplification simplifiée au déroulé narratif. L'intérieur d'un palais, d'une chambre, d'une galerie, d'un cabinet sont les formes visibles de ces "décor-décorum". La métonymie a changé : la forme iconographique du décor a pour référence une seule entité conceptuelle inscrite dans le champ visuel unifié perceptible par le spectateur. Le décor se charge ainsi d'une valeur apodictique : un palais ou une chambre à coucher, qui prend sa place dans toute l'étendue de l'aire scénique, s'impose à la vue du spectateur: les unités de lieu et de temps (et parfois aussi d'action) ont un support iconographique unique. Le "décor-décorum" se rattache de cette manière davantage à l'écriture régulière.

En revanche, le "décor-objet", qui caractérise selon nous la feinte irrégulière de l'Hôtel de Bourgogne avant 1640, s'applique davantage à une esthétique de la variété des lieux et des actions. Son utilisation spécifique dans la poétique irrégulière permet me semble-t-il de faire entrer le décor de théâtre de cette époque dans la notion d'objet dans sa caractéristique première que nous avons mis en exergue au début de l'article. La structure décorative n'est pas générique mais spécifique à une action plus ou moins isolée: elle possède une identité, une signification lors de l'évocation de l'action et elle n'a que peu de significations visuelles en dehors de cette action et de cette énonciation. Voyons quelques exemples. Plusieurs feintes de l'Hôtel de Bourgogne représentent sur scène des architectures de fontaines particulièrement imposantes (fig. 3) : elles sont composées de concrétions en étagement surmontées d'un entablement. Ces décors, en nombre limité dans les feintes (4 sur 47), sont très présents d'un point de vue architectural et donc visuel. La lecture des œuvres fait apparaître que ces fontaines ont un rôle très réduit dans le déroulement de l'action théâtrale. Les didascalies internes n'évoquent que des ruisseaux ou des ondes qui n'ont rien à voir avec les architectures visibles sur scène. Il est difficile d'imaginer que cette disposition sur l'aire de jeu ne soit qu'une volonté d'occuper l'espace. Par ailleurs, il est inenvisageable de croire à une simple répétition schématique d'un décor. Il semblerait que cette présence se rattache davantage à une "actualité" architecturale.

Dans les années 1620-1630, la construction du palais de Marie de Médicis, aujourd'hui le palais du Luxembourg, occupe en effet tous les esprits. La Reine fait bâtir, à l'image des nymphées des jardins italiens, la "grotte" du Luxembourg, dénommée depuis lors «Fontaine Médicis " ${ }^{14}$. Tommaso Francini, Intendant Général des Eaux et Fontaines royales, en aurait dessiné les plans. Il a, par ailleurs, conçu et réalisé plusieurs architectures de ce type, toutes font appels au détail de la concrétion minérale comme chez Buffequin. Ainsi, la fontaine architecturée représentant un « ruissellement minéral », qui se confond quelque peu avec la grotte, fait partie d'un horizon contemporain pour le spectateur du début du XVIIe siècle ; d'où, semble-t-il, la place prépondérante qu'elle occupe dans les esquisses de Buffequin. Loin d'être un simple ornement décoratif, cette fontaine marque la référence à une actualité architecturale du début du XVIIe siècle.

14 La fontaine du XVIIe siècle ne possédait pas de sculpture et la niche était vide. Cette fontaine avait été commandée par Marie de Médicis et était destinée à fermer la perspective de l'allée transversale est-ouest qui passait devant la façade du palais. Avec les aménagements urbains d'Hausmann, la fontaine fut déplacée et on lui ajouta les sculptures présentes aujourd'hui. 
L'image du décor constituerait un vecteur d'information et de diffusion d'une actualité artistique. En l'occurrence il s'agit ici d'une création architecturale récente liée à la reine. Mais d'autres parties des décors de Buffequin présentent ce caractère informatif qui concernent généralement des détails de la vie quotidienne (enseigne ou structure de boutique: marchand d'art, boucher) ou d'éléments architecturaux (fontaines, façades de palais, ornements architecturaux). La feinte théâtrale irrégulière, loin de former un simple support d'action, transmet une information qui offre la possibilité au spectateur de rattacher les éléments du décor à son propre quotidien. Notons au passage que certaines de ces fontaines, font, parfois, réellement jaillir de l'eau. Si l'on considère de surcroît que les spectateurs pouvaient entendre le chant des rossignols sifflé depuis les coulisses, on imagine aisément l'effet rendu.

La fonction symbolique du décor, comme catégorie de l'objet, se retrouve également dans la représentation de la prison. Lieu d'enfermement, la geôle, malgré sa faible figuration dans les différents dessins de Buffequin, est très visible quand elle apparaît. Elle est un lieu qui s'oppose à l'amour et à l'union des êtres; elle préfigure une exécution capitale (fig. 2), mais elle est toujours le lieu du dialogue ou du monologue, de la plainte d'un amour contrarié, ce qui explique son caractère praticable : le héros se situe à l'intérieur de la feinte, derrière des barreaux, dans une certaine obscurité. Un examen attentif des dessins montre que Buffequin sait diversifier la représentation de cette feinte : elle peut être de forme carrée, grande (fig. 4) ou petite (fig. 5), elle est parfois placée dans un bâtiment large ou, enfin, elle a une forme ronde avec ou sans porte d'accès sur scène (fig. 2). Malgré sa faible importance quantitative dans le déroulement de chaque action dramatique, le décor de la prison prend une importance plastique, venant souligner de la sorte un moment particulièrement tragique.

Comme l'a montré Jürgen Habermas, les notions d'espace public et d'espace privé n'émergent dans leur définition actuelle qu'au XVIIIe siècle ${ }^{15}$. Toutefois, on peut constater que le décor irrégulier propose des zones symboliques de dramaturgie qui délimitent des entités spécifiques qui seront, soit liées à l'intime (la chambre, le jardin, la prison, autant d'images scéniques favorables au dialogue entre peu de personnages); soit, au contraire, propice à l'extériorité (la place publique, la salle de trône, le château, éléments dramaturgiques favorables à l'action avec plusieurs personnes). L'espace scénique irrégulier se décompose donc en champs narratifs singuliers qui renvoient chacun à un imaginaire de la répartition sociale et poétique spécifique. Néanmoins, il semble difficile de considérer ce théâtre irrégulier comme étant déjà l'exercice de décor autonome suggérant par leur présence une valeur intrinsèque. C'est le théâtre unifié par les règles aristotéliciennes plus tardif du XVIII siècle qui portera cette valeur objectivante du décor.

Ainsi, dans l'esthétique irrégulière, certains décors prennent une grandeur (occupation de l'espace et utilisation scénique) proportionnelle à l'intensité dramatique. La répartition décor-objet a eu pour délimitation traditionnelle la taille de l'entité : au décor reviendrait la grandeur, tandis qu'à l'objet serait attribué la petitesse. Cette répartition n'a pas tout à fait sa pertinence dans le théâtre irrégulier.

15 Jürgen Habermas, L'Espace public. Archéologie de la publicité comme dimension constitutive de la société bourgeoise, Paris, Payot, 1993. 
Comme je l'ai montré, un décor imposant sur scène par la taille n'a finalement qu'une petite place dans l'économie globale de la dramaturgie, et un objet de petite taille peut devenir l'enjeu principal de la pièce (par exemple La Bague de l'oubli de Rotrou). La fonction métonymique des décors irréguliers au début du XVIIe siècle prend son origine historique dans le mystère médiéval où chaque élément décoratif est présent simultanément sur scène et où chaque partie peinte renvoie à un univers dramaturgique plus ample. L'hôtel de Bourgogne ayant d'ailleurs servi de lieu de représentation depuis le milieu du XVIe siècle pour la Confrérie de la Passion célèbre pour ses mystères sacrés.

De cette manière, l'idée de "décor-objet", propre à l'esthétique théâtrale du début du XVIIe siècle, offre la possibilité de définir une catégorie scénique qui finalement met en interpénétration l'objet et le décor, aussi bien dans sa qualité quantitative que poétique. Cette porosité des catégories constitue une caractéristique de cette période.

Certaines feintes du début du XVIIe siècle, par cette combinaison de fragments et de détails, entre actualité et symbolisme, construisent un imaginaire propre au spectateur de cette époque. Un regard rapide sur ces dessins pourrait n'y voir qu'une extravagance graphique sans lien avec la réalité artistique ou sociale. Et si on constate que le décor s'apparente à un signifiant symbolique, il faut toutefois ne pas négliger qu'il est aussi, et en même temps, une démonstration, une citation directe. Le décor théâtral irrégulier ne procède pas uniquement par ellipse, sous-entendu ; il est également une référence immédiate, un langage non-ambivalent, sans détour. La fragmentation des univers urbains, la dissémination des significations, la complexité des images de décor et de leurs influences sont autant de critères d'analyse présents dans ces esquisses. L'approche iconographique est alors une manière de constater l'enchevêtrement référentiel dans l'élaboration d'une feinte théâtrale. Elle ne se construit pas nécessairement sur des références mythologiques, théologiques, poétiques ou philosophiques habituelles. Elle s'invente d'après des "citations" plus ou moins directes en rapport avec une actualité et un univers de formes et de connaissances partagé par le plus grand nombre, ou alors elle invente un langage visuel propre, performatif et non réaliste mais particulièrement signifiant pour le spectateur.

Le décor dans l'esthétique de la variété peut donc s'assimiler à un objet, solide, «maniable, généralement fabriquée, un et indépendant (dans son utilisation singulière mais pas nécessairement dans sa valeur symbolique), ayant une identité propre, qui relève de la perception extérieure, appartient à l'expérience courante et répond à une certaine destination ». Ici, les notions de perception et d'expérience courante nous semblent fondamentales pour désigner ces décors irréguliers de Buffequin comme étant des objets. Ils ne sont pas des décors neutralisés comme pour le «palais à volonté ». Ce "décor-décorum" là n'appartient pas à la catégorie de l'objet car il n'est pas performatif : il n'acquiert sa signification qu'en étant le support, l'arrière fond visuel, le décoratif de l'action. Dans le "décor-décorum", l'action scénique (surtout dans la tragédie) ne qualifie presque jamais le cadre décoratif dans lequel se déroule l'action. La caractéristique du lieu est en effet très souvent donnée en début d'action, en didascalie externe («la scène se situe dans un 
palais ») et au cours du déroulement de la pièce il est très rare que l'identité du lieu n'intervienne dans l'oralité poétique.

En revanche, certains décors de l'esthétique irrégulière sont des objets : ils deviennent visibles et identifiables en même temps que le déroulé d'une action spécifique. Ils répondent à une destination précise au moment de l'action dramaturgique et ils sont perçus comme signifiant au moment du jeu des acteurs durant un laps de temps déterminé. La poétique irrégulière se caractérise de cette manière par la nécessité pour les acteurs - en raison de la simultanéité des décors visibles sur scène - d'énoncer le cadre décoratif dans lequel leur action se poursuit. Ce décor est en cela performatif car il est identifiable par le spectateur à partir du moment où l'acteur, en didascalie interne, explicite clairement le décor dans lequel évolue son action. En cela, le "décor-objet" s'incarne grâce à l'énonciation poétique. L'action n'est pas suffisante, il y faut également une explication de texte. C'est notamment ce dernier aspect du décor irrégulier qui a choqué les partisans de l'écriture régulière, le besoin pour l'acteur de toujours énoncer le décor dans lequel il évolue afin que le spectateur puisse comprendre le déroulé dramatique, et ceci d'autant plus que plusieurs décors différents pouvaient être présents sur scène à l'arrière plan du comédien.

\section{La lumière comme immatérialité réelle du théâtre irrégulier}

D'autres objets constituent les scénographies préclassiques de l'Hôtel de Bourgogne. Ces entités ont cependant une caractéristique particulière : elles sont visibles par le spectateur, elles ont même un rôle important dans la dramaturgie, et pourtant elles demeurent sans matérialité. Ce sont des objets sans contours déterminés et pourtant ils ont une délimitation observable. Ils sont ce que je propose d'appeler des "immatériels réels". En examinant leur consistance, je propose d'élargir le champ de la notion d'objet: non pas uniquement des structures matérielles qui viennent appuyer ou accompagner une action ou un verbe scénique, mais aussi des entités immatérielles, au contour plus ou moins défini, mais qui sont observables par le spectateur, et qui acquièrent par la même le statut de représentation, de mise en présence d'une réalité mais qui n'est pas matérielle. Ces objets immatériels sont des constructions volontaires, des élaborations dramaturgiques voulues par les scénographes, ils sont donc le fruit d'une pensée, et pourtant ils demeurent sans lignes de contours définies. La lumière scénographique me semble faire partie de ces objets immatériels.

Les acteurs et les spectateurs du théâtre de l'Hôtel de Bourgogne apprécient particulièrement les nuances lumineuses sur scène (cf. liste ci-jointe), et d'une manière générale durant la première partie du XVIIe siècle ${ }^{16}$. Que ce soit dans la fabrication des nuits ou bien dans la présence sur scène de bougeoirs, de torches ou même de flammes, les spectateurs aiment le clair-obscur scénique. Le maître des

16 Voir notamment: Paulette Choné, L'atelier des nuits. Histoire et signification du nocturne dans l'art d'Occident, Nancy, Presses universitaire de Nancy, 1992 ; Paulette Choné, Jean-Claude Boyer, Richard E. Spear, Irving Lavin [dir.], L'Âge d'or du nocturne, Paris, Gallimard, 2001. 
feintes va jusqu'à prévoir la venue sur scène d'un feu d'artifice (fig. 6), sans que l'on sache si un tel décor crachait réellement des flammes...

En observant attentivement les dessins, on constate que plusieurs des décors possèdent une ombre portée. Les compartiments, côté jardin, sont presque toujours grisés et une ombre se projette sur le plancher. Cette ombre indique le placement de la source lumineuse : dans les cintres côté jardin. C'est cette même place que Nicolo Sabbatini préconise : «donc, suivant et notre opinion et l'opinion commune, nous désignons comme la plus goûtée cette façon de peindre les scènes en faisant venir ainsi la lumière ${ }^{17}$ ». L'expression de Sabbatini, «l'opinion commune » est importante, elle signifie que cette pratique est inscrite dans une coutume théâtrale italienne ancienne, mais également française, puisqu'elle est présente à l'Hôtel de Bourgogne. Les remarques de Jacques Scherer expliquent les raisons d'un tel placement de la lumière : «Dans toutes les civilisations, [...], la droite est le côté du bonheur et du sacré, tandis que la gauche est exclue et porte malheur [...]. Or, dans tous les exemples que nous connaissons, sans aucune exception, des représentations théâtrales du Moyen Âge, c'est le contraire qui se produit: pour le spectateur, l'enfer est à droite et le paradis à gauche ». Dans les décors de Buffequin, la lumière vient de la gauche du spectateur. Jacques Scherer rajoute : «Devant le scandale du paradis à gauche du spectateur, il faut chercher, dans l'espace double des mystères, un être qui aurait, comme il se doit, le paradis à sa droite. Cet être ne peut être que Dieu. [...] Dieu est nécessairement situé là où il peut voir le paradis à sa droite et l'enfer à gauche ${ }^{18}$ », c'est-à-dire au niveau du front de scène, à l'emplacement du point de fuite.

L'absence de la lumière sur scène, produisant une demi-obscurité (la salle reste illuminée par des chandelles) est tout aussi importante. À partir de la liste des accessoires de Buffequin, il est possible en effet de calculer qu'il y a au moins un tiers de nuits qui sont utilisées dans les pièces de l'Hôtel de Bourgogne. Ces nuits semblent avoir été obtenues par l'obstruction des différentes sources lumineuses : soit de manière définitive par l'extinction des lumières naturelles (bougies, lampes à huile), soit momentanée grâce à des caches placés devant les points lumineux. Ainsi, les décès de Pyrame et de Thisbé (fig. 7) ne pourraient atteindre l'apogée tragique sans une obscurité voulue par la mise en scène. La lumière, par sa présence (ou son absence), qualité souvent précisée par les comédiens, joue donc, à l'identique du décor, un rôle actif.

En outre, le public est également relié au monde de la représentation par la lumière car la plupart des représentations théâtrales se déroule dans une salle lumineuse. Sabbatini, au XVIIe siècle, précise : «qu'on fasse les flambeaux en considération de ceci, c'est-à-dire gros et courts, suffisants toutefois, pour durer tout le temps de la comédie, et il n'y aura, alors danger qu'ils ployent, ni même coulent de trop $^{19} »$ sur le public. Avec les indications de Sabbatini, l'éclairage «tout le

\footnotetext{
17 Nicolo Sabbatini, Pratica di fabricar scene e machine ne' teatri, Pesaro, 1637, Louis Jouvet (trad.), Pratique pour fabriquer scènes et machines de théâtre, Neuchâtel, Ides et Calendes, 1942. 2e édition 1994, p. 22.

18 Jacques Scherer, «Métamorphoses de l'espace scénique», dans Le Théâtre en France, Jacqueline de Jomaron [dir.], Paris, Armand Colin, 1992, 2e éd., t. 1, p. 132-133.

19 Nicolo Sabbatini, Pratica di fabricar scene..., op. cit., livre I, Chapitre 38, p. 63 de l'édition de 1994.
} 
temps de la comédie » semble visible en permanence dans la salle pendant les représentations. Cette idée est reprise et développée par André Villiers : l'unité entre la salle et la scène s'établit grâce à la présence de la lumière de part et d'autre. «L'union de la salle et de la scène est une réalité physique »; et il conclut : «pour le spectateur du XVIIe siècle, la salle et la scène ne sont pas séparées tant que dure le spectacle; il n'y a pas de coupure entre le monde illusoire et le monde réel, fondus ensemble dans la théâtralité du cérémonial ${ }^{20}{ }$.

Enfin, la place de la nuit dans la règle des vingt quatre heures joue un rôle important dans la mise en place de la théorie de l'unité de temps. Pour ne pas rendre trop rigide cette unification, plusieurs auteurs des années 1630 vont considérer que le temps narratif pouvait durer vingt-quatre heures (et non durant le temps de la représentation) et qu'ainsi le déroulement dramaturgique avait la possibilité d'accueillir une nuit. Cette "pause", placée généralement au centre de la pièce, apportait ainsi une césure dans l'action dramatique et permettait une moindre rigidité de la règle des vingt-quatre heures, créant de cette manière un rythme narratif différent entre le diurne et le nocturne.

La lumière scénique joue donc un rôle important dans l'esthétique régulière et dans l'art de la variété. Au delà de sa fonction de mise en avant ou pas de l'action, elle apparaît comme une entité propre, soutenant à la fois l'action des comédiens, mais également les décors. Elaborée avec science, la question de l'éclairage et de ses effets sur scène a été l'enjeu d'une politique artistique ambitieuse de Richelieu: quand il décide d'ouvrir la première grande salle à l'italienne dans son Palais, il fait tout son possible pour faire venir Le Bernin d'Italie. L'architecte-sculpteur de la famille Barberini est reconnu pour ses inventions mais aussi pour ses scénographies, en particulier dans la technique du coucher du soleil sur scène. Si le romain refuse de venir à Paris (voyage qu'il effectue tout de même en 1665 pour Louis XIV), il envoie néanmoins un de ses assistants, Gian Maria Mariani, spécialiste des effets de machinerie, qui effectue le déplacement pour créer le mouvement du soleil dans Mirame, pièce qui inaugure en janvier 1641 le grand théâtre du palais cardinal ${ }^{21}$. On remarque ainsi que la question de la lumière scénique, dans les années 1630-1640, au moment où Buffequin réalise des décors pour l'Hôtel de Bourgogne et pour Richelieu, est un enjeu à la fois esthétique et politique d'une réelle importance.

La lumière sur scène est donc le produit d'objets de combustion (bougies, mèches...) mais produit un effet "immatériel": elle crée du sens poétique et esthétique, elle est un acteur à part entière de la dramaturgie comme on constate dans les deux listes citées plus haut. En reprenant la définition de la notion d'objet, on peut donc dire que la lumière est «maniable, généralement fabriquée, une et indépendante, ayant une identité propre, qui relève de la perception extérieure, appartient à l'expérience courante et répond à une certaine destination ». Elle n'est pas générée par un objet solide (la flamme), mais sa construction, son effet, sa

\footnotetext{
20 André Villiers, «Illusion dramatique et dramaturgie classique », XVIIe siècle, $\mathrm{n}^{\circ}$ 73, 1966, pp. 3-35.

Marc Bayard, «Le roi au cœur du théâtre... », art. cit.; Benoît Bolduc, «Stefano Della Bella, inventeur des gravures des "Nozze degli dei" (1637) et de "Mirame" (1641) », Marc Bayard [dir.], Rome-Paris, 1640. Transferts culturels et naissance d'un centre artistique, Rome-Paris, Académie de France à Rome-Somogy, 2010, p. 481-507.
} 
perception et sa destination lui donne toutes les caractéristiques de l'objet, non pas matériel, mais immatériel redéfinissent sur de nouvelles bases (rationnelles, physiques) le réel.

L'esthétique irrégulière de l'art dramatique des années 1620-1640 a donc une spécificité poétique, mais aussi, comme je l'ai montré, une particularité scénographique dans sa manière de composer l'enchaînement des décors. En jouant de la qualité solide des objets (accessoires mobiles), en utilisant la feinte comme un objet (le "décor-objet" et sa singularité performative), Buffequin élargit sa fabrication de l'art scénographique en façonnant des objets "immatériels réels" que constitue l'éclairage scénique.

L'esthétique irrégulière joue des ambivalences des règles poétiques et de ses composantes matérielles (décors, accessoires, effets) que la poétique unitaire a réussi à bannir au nom de la cohésion, de la vraisemblance et de la convenance.

\section{Bibliographie}

BAYARD Marc, «Les faiseurs d'artifices: Georges Buffequin et les artistes de l'éphémère à l'époque de Richelieu », Revue XVIIe siècle, n² 230, 1-2006, p. 151164.

BAYARD Marc, "Le roi au cœur du théâtre : Richelieu met en scène l'Autorité », L'image du roi de François Ier à Louis XIV, Thomas W. Gaehgtens et Nicole Hochner [dir.], Actes de colloque 2001, Centre Allemand d'histoire de l'art/Éditions de la Maison des sciences de l'homme, Paris, 2006, p. 191-208.

BAYARD Marc, «Les dessins du Mémoire de Laurent Mahelot : sur les traces d'un peintre du roi au service de Richelieu », Revue d'histoire du théâtre, 2006-4, p. 313324.

BAYARD Marc, «Richelieu en scène, Buffequin en coulisse », Richelieu, patron des arts, J.-C. Boyer, B. Gaehtgens et B. Gady [dir.], Paris, Éditions de la Maison des Sciences de l'homme, 2009, p. 475-500.

BAYARD Marc, Feinte Baroque. Iconographie et esthétique de la variété au XVIIe siècle, Rome/Paris, Académie de France à Rome/Somogy, 2010.

BAYARD Marc, «De la cohérence à la cohésion: quelques réflexions sur la construction de la théorie de l'unité dans les arts en Italie et en France (XVIe-XVIIe siècles) », Marc Bayard [dir.], Rome-Paris, 1640. Transferts culturels et renaissance d'un centre artistique, Rome/Paris, AFR/Somogy, 2010, p. 415-431

BLOCKER Déborah, Instituer un "art" : politiques du théâtre dans la France du premier XVIIe siècle, Paris, Champion, 2009.

BOLDUC Benoît, "Stefano Della Bella, inventeur des gravures des "Nozze degli dei" (1637) et de "Mirame" (1641)», Marc Bayard [dir.], Rome-Paris, 1640. Transferts culturels et naissance d'un centre artistique, Rome-Paris, Académie de France à Rome-Somogy, 2010, p. 481-507.

CHONÉ Paulette, L'atelier des nuits. Histoire et signification du nocturne dans l'art d'Occident, Nancy, Presses universitaire de Nancy, 1992.

CHONÉ Paulette, BOYER Jean-Claude, SPEAR E. Richard, LAVIN Lavin [dir.], L'Âge d'or du nocturne, Paris, Gallimard, 2001.

CIVARDI Jean-Marc, La querelle du Cid.1637-1638, Paris, Champion, 2004. 
Dictionnaire CNRTL: URL : http://www.cnrtl.fr/definition/[site consulté le 31/10/2011].

DOTOLI Giovanni, Temps de préfaces : le débat théâtral en France de Hardy à la querelle du "Cid", Paris, Klincksieck, 1997.

DUPRAT Anne, Vraisemblances. Poétiques et théorie de la fiction du Cinquecento à Jean Chapelain (1500-1670), Paris, H. Champion, 2009.

FRAISSE Simone, Une conquête du rationalisme, l'influence de Lucrèce en France au XVIe siècle, Paris, Nizet, 1962.

GAMBINO LONGO Susanna, Savoir de la nature et poésie des choses. Lucrèce et Épicure à la Renaissance italienne, Paris, Champion, 2004.

Greimas, Algirdas Julien, Sémantique structurale : recherche et méthode, Paris, Larousse, 1966.

Jürgen, L'Espace public. Archéologie de la publicité comme dimension constitutive de la société bourgeoise, Paris, Payot, 1993.

HENIN Emmanuelle, Ut pictura theatrum: théâtre et peinture de la Renaissance italienne au classicisme français, Genève, Droz, 2003.

HENIN Emmanuelle [dir.], Les Querelles dramatiques à l'Age classique (XVIIeXVIIIe siècles), Louvain, Peeters, coll. La République des Lettres 40, 2010.

LESTRINGANT Frank [dir.], La renaissance de Lucrèce, Paris, PU Paris-Sorbonne, Collection Cahiers V.L. Saulnier, 2010.

PASQUIER Pierre (éd.), Le Mémoire de Mahelot, Laurent et d'autres décorateurs de l'Hôtel de Bourgogne et de la Comédie-Française au XVIIIe siècle, Paris, Champion, 2005.

SABBATINI Nicolo, Pratica di fabricar scene e machine ne' teatri, Pesaro, 1637, Louis Jouvet (trad.), Pratique pour fabriquer scènes et machines de théâtre, Neuchâtel, Ides et Calendes, 1942. 2e éd. 1994.

SCHERER Jacques, "Métamorphoses de l'espace scénique », dans Le Théâtre en France, Jacqueline de Jomaron [dir.], Paris, Armand Colin, 1992, 2e édition, t. 1, p. 132-133.

VILLIERS André, «Illusion dramatique et dramaturgie classique », XVIIe siècle, $\mathrm{n}^{\circ}$ 73, 1966, p. 3-35.

\section{Liste des illustrations}

fig. 1 - Georges Buffequin, dessin du décor des Occasions perdues, tragicomédie de Rotrou. Paris, Bibliothèque nationale de France, Ms. Fr. 24330, $\mathrm{f}^{\circ}$ 
11

$\mathbf{r}^{\circ}$

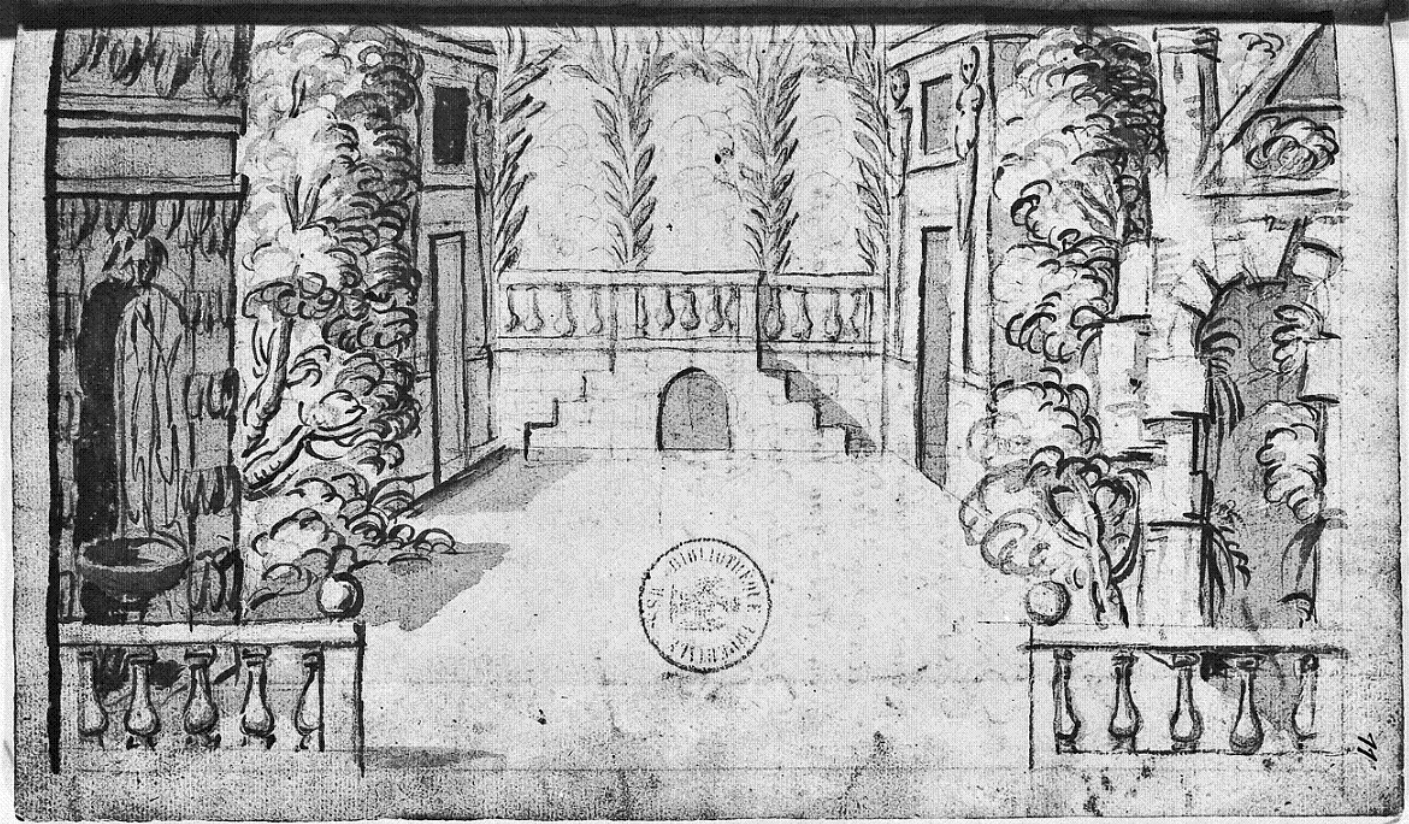

fig. 2 - Georges Buffequin, dessin du décor de Clitophon, tragi-comédie de Du Ryer. Paris, Bibliothèque nationale de France, Ms. Fr. 24330, $f^{\circ} 48 \mathbf{r}^{\circ}$.

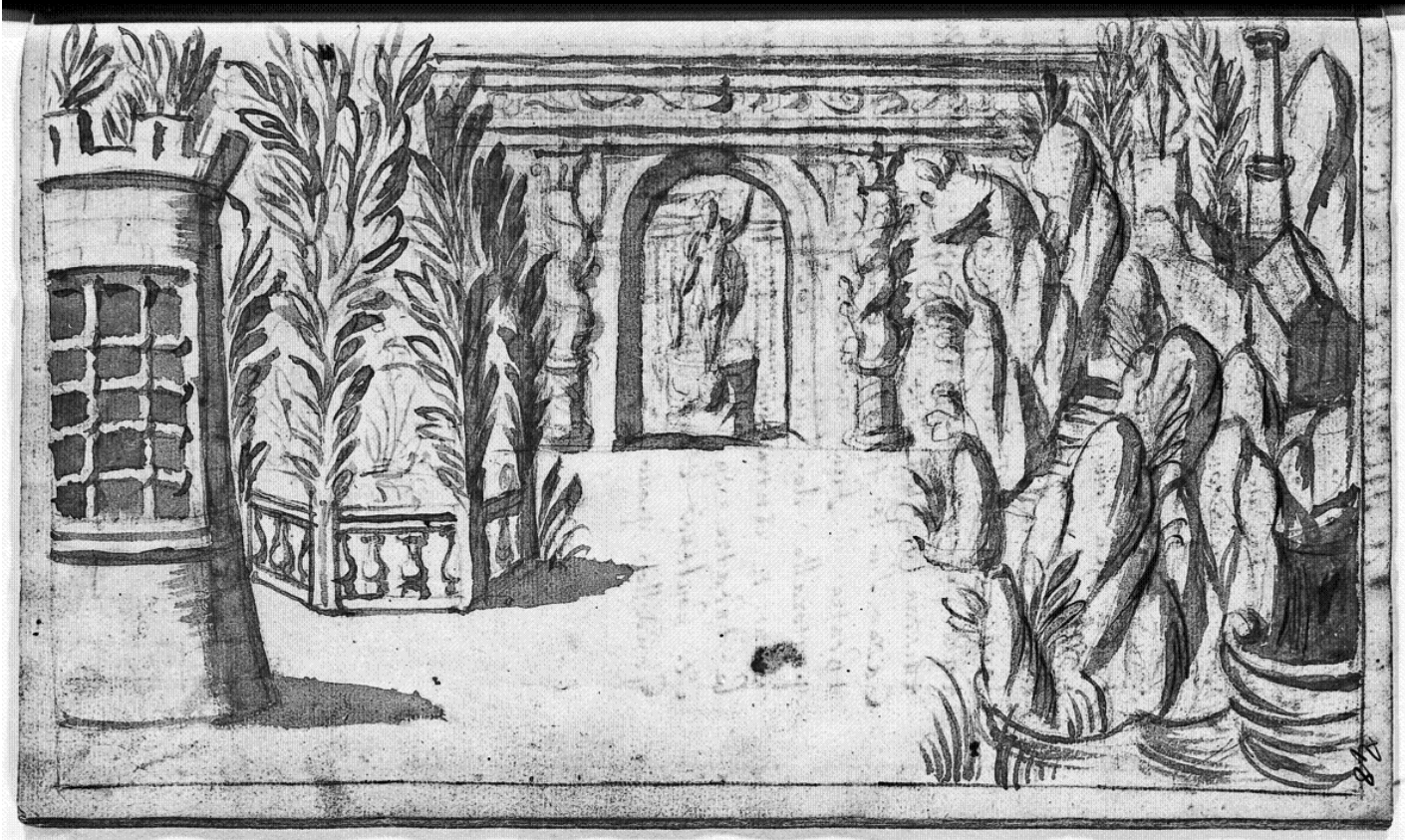


fig. 3 - Georges Buffequin, dessin du décor de La Filis de Scire, pastorale de Pichou. Paris, Bibliothèque nationale de France, Ms. Fr. 24330, $f^{\circ} 46 r^{\circ}$.

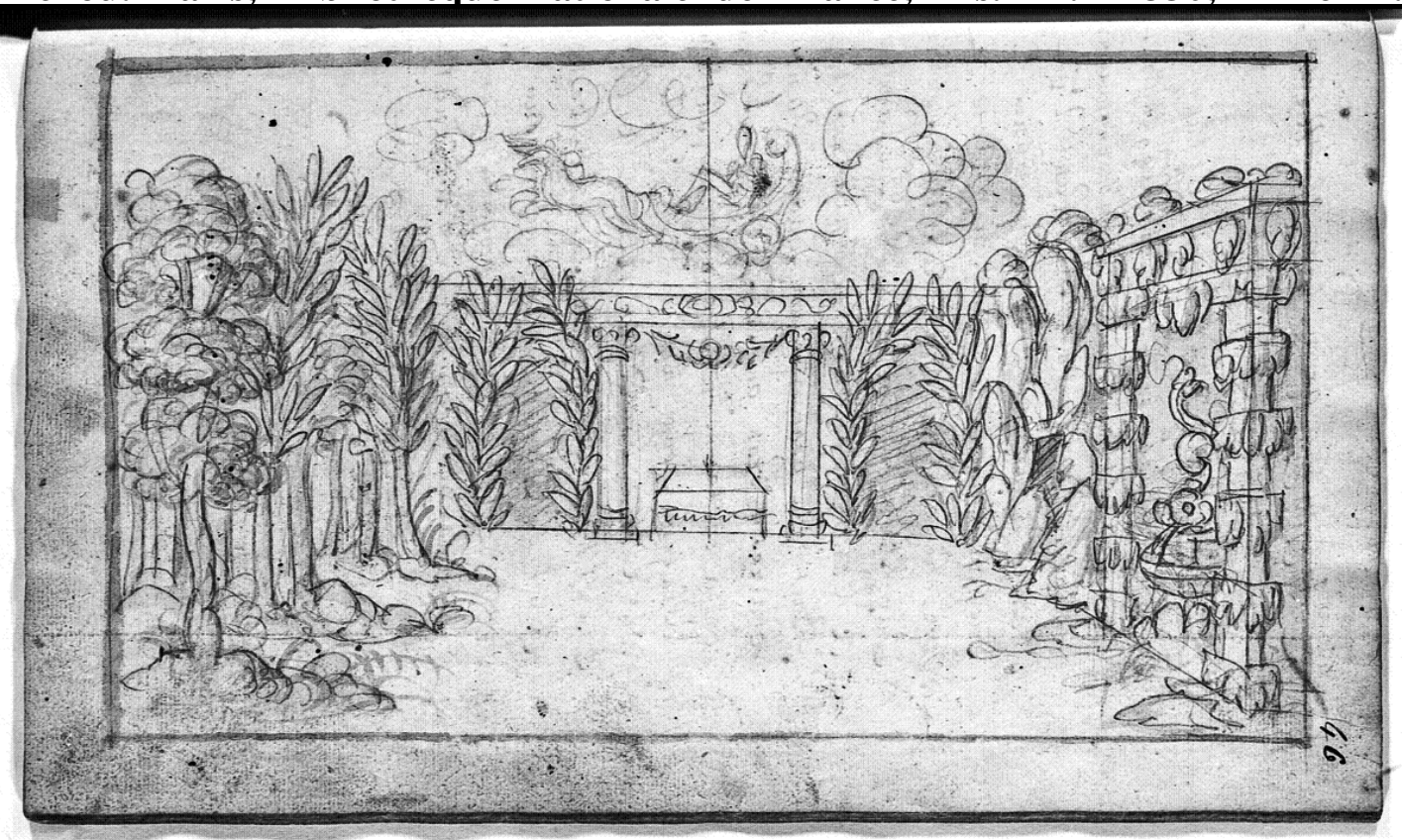

fig. 4 - Georges Buffequin, dessin du décor de Pandoste (1er journée) de Hardy. Paris, Bibliothèque nationale de France, Ms. Fr. 24330, $\mathbf{f}^{\circ} \quad 21 \quad \mathbf{r}^{\circ}$.

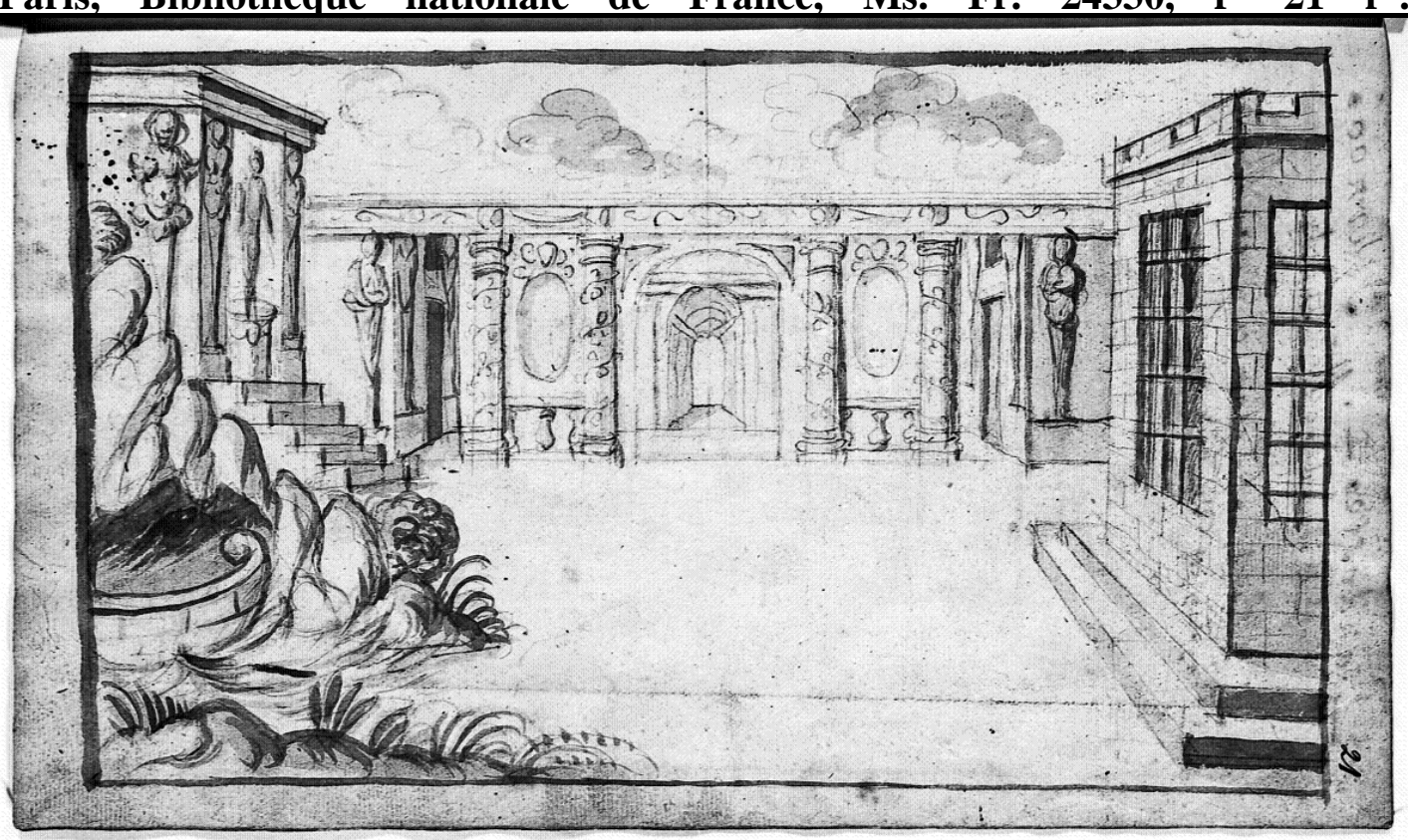

fig. 5 - Georges Buffequin, dessin du décor de Ligdamon et Lidias, tragicomédie de Scudéry. Paris, Bibliothèque nationale de France, Ms. Fr. 24330, $\mathbf{f}^{\circ}$ 


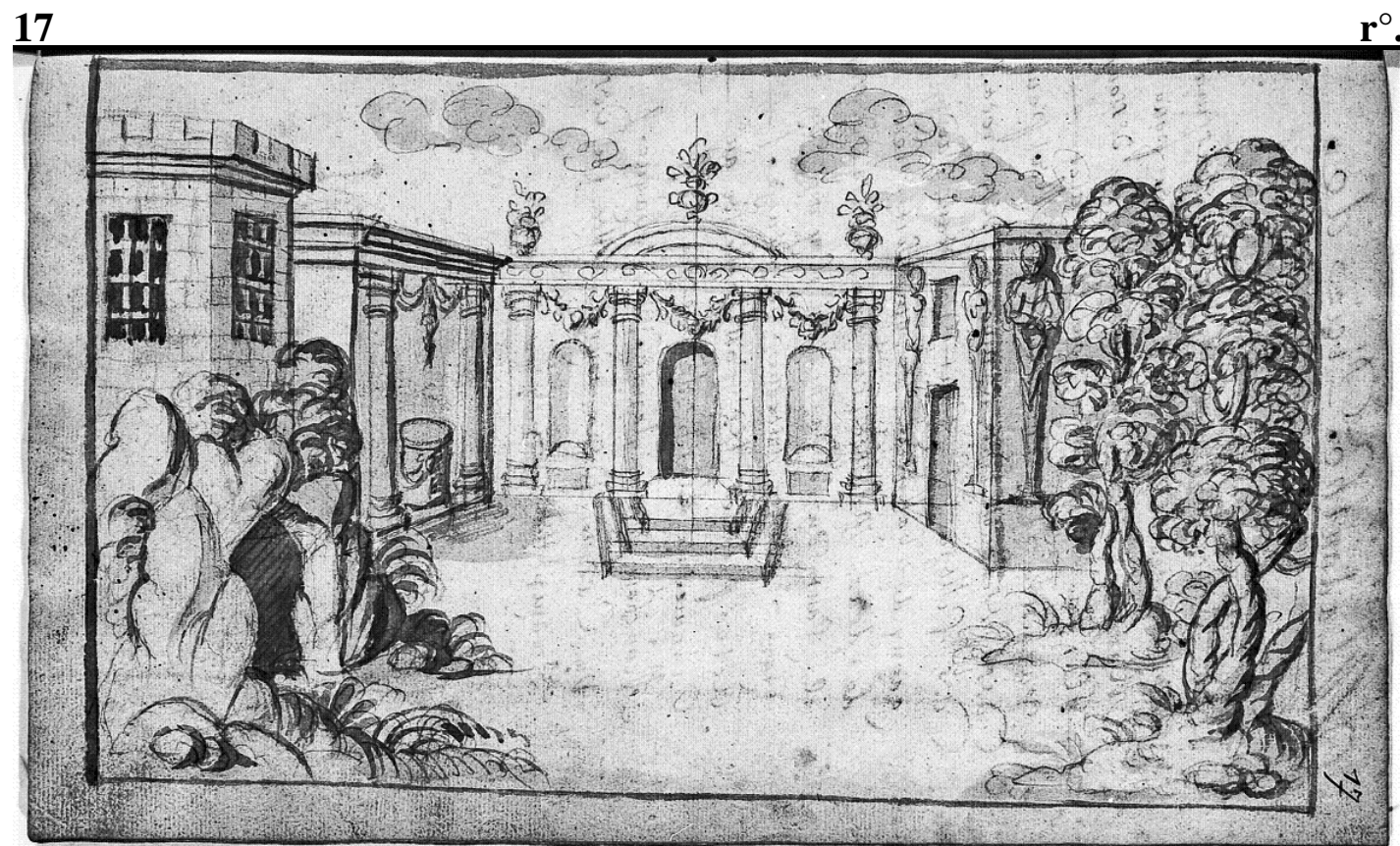

fig. 6 - Georges Buffequin, dessin du décor de l'Argénis, tragi-comédie de Du Ryer. Paris, Bibliothèque nationale de France, Ms. Fr. 24330, $f^{\circ} 38 \mathbf{r}^{\circ}$.

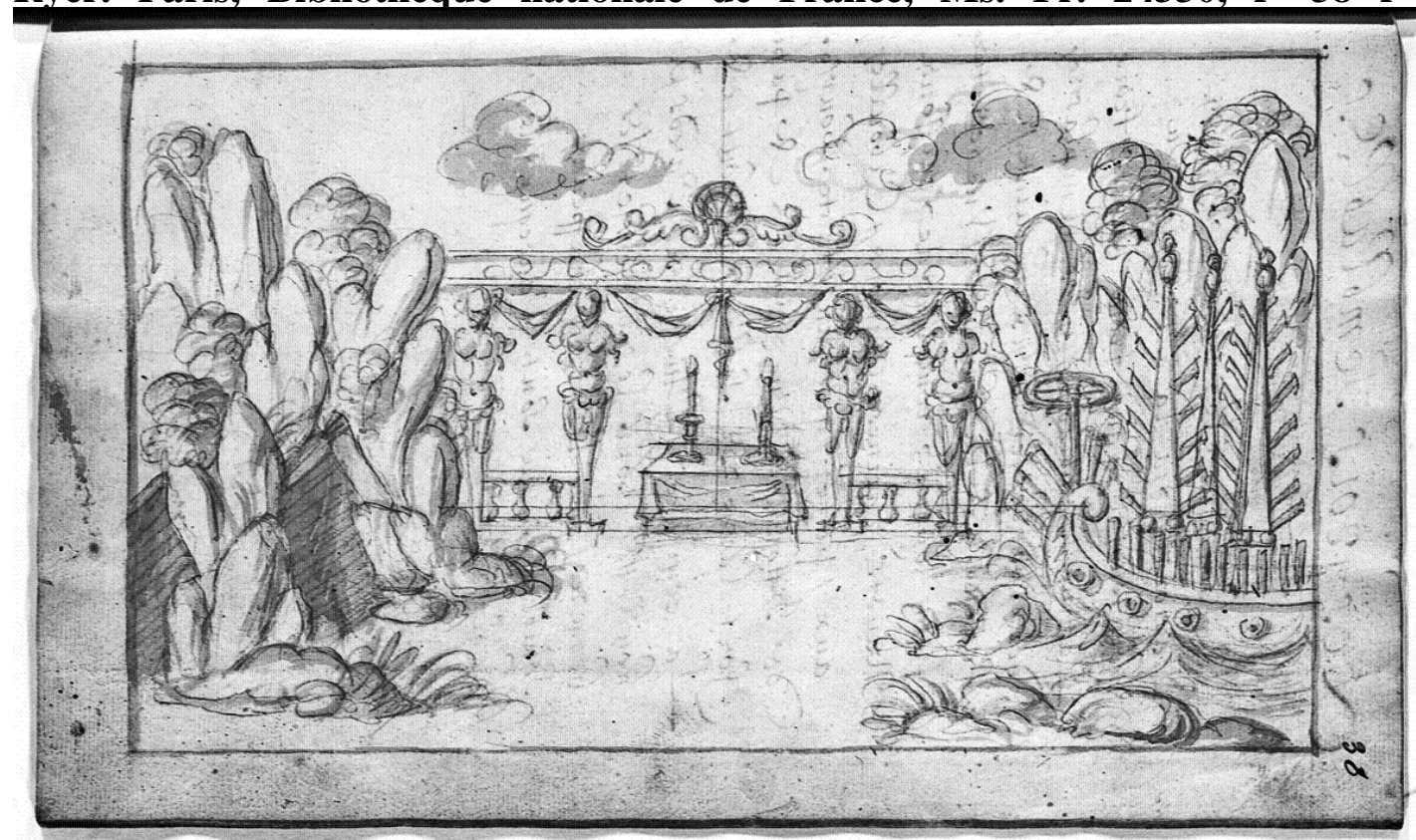

fig. 7 - Georges Buffequin, dessin du décor de Pyrame et Thisbé, tragédie de Théophile de Viau. Paris, Bibliothèque nationale de France, Ms. Fr. 24330, $\mathbf{f}^{\circ}$ 


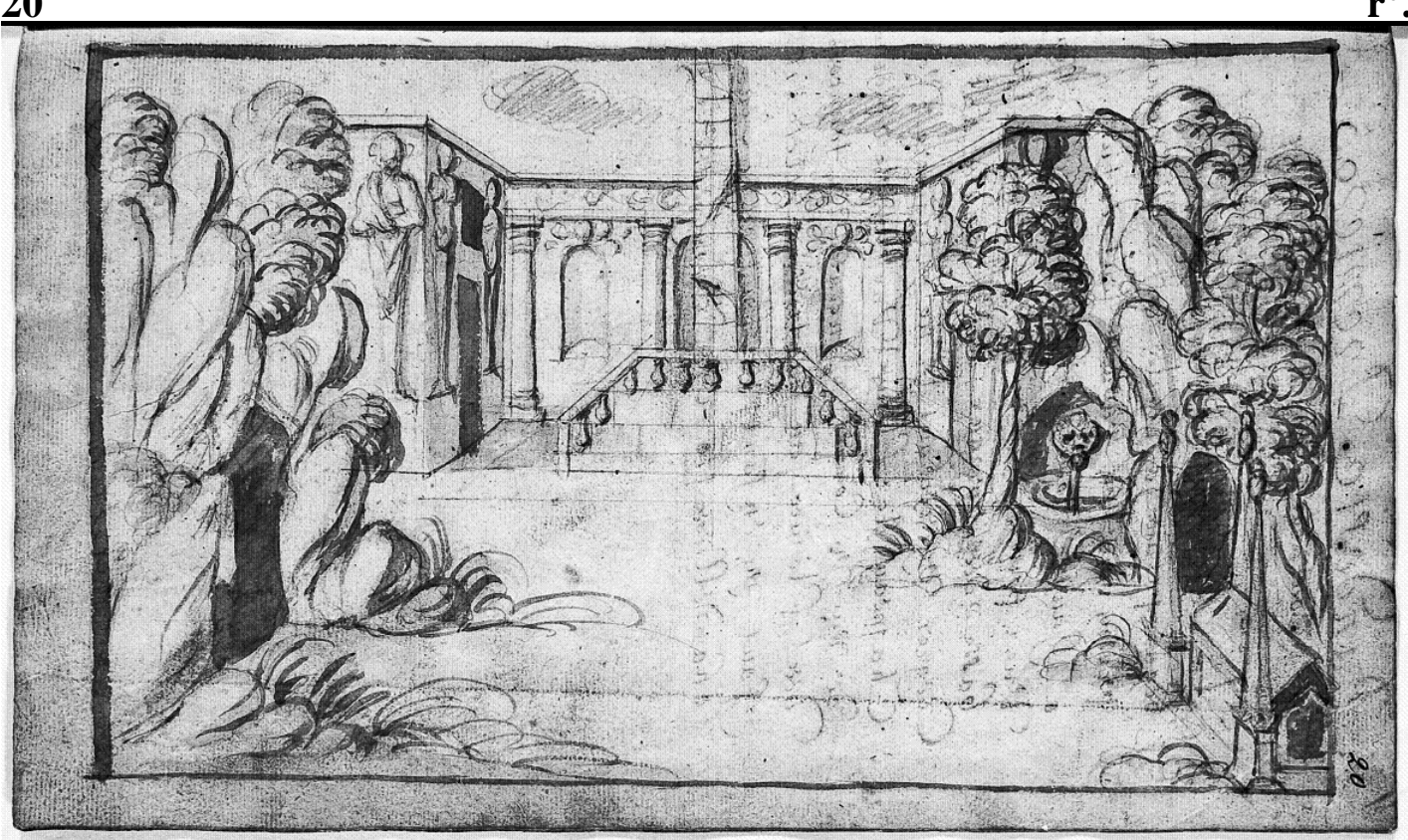

\section{Pour citer le document}

Marc Bayard, «Le décor comme objet dans l'esthétique théâtrale irrégulière du XVIIe siècle», Agôn [En ligne], Pour une archéologie de l'objet théâtral, $\mathrm{N}^{\circ} 4$ : L'objet, Dossiers, mis à jour le : 14/01/2012, URL : http://agon.enslyon.fr/index.php?id=2024 